\title{
Değişken gecikmeli kısıtlı stokastik kontrol geçiş sistemleri için regülatör problemi
}

\author{
Çerkez AĞAYEVA ${ }^{1,2 *}$ \\ ${ }^{1}$ Muş Alparslan Üniversitesi, Ikktisat Bölümü,49250, Muş \\ ${ }^{2}$ Institute of Control, Azerbaijan National Academy of Science, Baku \\ (ORCID: 0000-0003-0507-9785)
}

\begin{abstract}
Öz
$\mathrm{Bu}$ makalede, doğrusal stokastik denklemler sınıfıyla ifade olunan geçiş sistemleri ele alınmıştır. Gecikmeli faz ve kontrol parametreleri içeren diferansiyel denklemler için karesel amaç fonksiyonu olan optimal kontrol problemi oluşturulmuş ve sağ uç noktasında kısıta sahip olan durum için optimizasyon problemi incelenmiştir. Literatürde Doğrusal Karesel Regülatör olarak bilinen ve sabit katsayılı stokastik diferansiyel denklemlerle ifade olunan bu problemin optimal lığı için yeter ve gerek koşul, maksimum prensibi şeklinde ispatlanmıştır. Bunun yanı sıra geçiş sistemleri için önemli olan geçiş noktalarının bulunması için karşıtlık koşulları bulunmuştur. Sonda ise Doğrusal Karesel Regülatör problemleri için önem taşıyan optmal kontrolün geri dönüşüm şekli bulunmuştur. Çözümü, Riccati denklemleriyle ifade olunan geri dönüşüm problemi, bu çalışmada değişken gecikmeli stokastik sistemler için uygulanmıştır.
\end{abstract}

Anahtar kelimeler: Optimal kontrol problemi, gecikmeli stokastik diferansiyel denklemler, geçiş sistemleri, Riccati denklemleri.

\section{Regulator problem of restricted stochastic switching systems with variable delay}

\begin{abstract}
In this paper, the switching systems expressed by the class of linear stochastic differential equations are discussed. For the differential equations containing the delayed phase and delayed control parameters, the optimal control problem with the quadratic cost function is constructed. Stochastic optimization problem is examined for the systems with the the right end point restriction. Linear Quadrature Regulator problem expressed by the stochastic differential equations is investigated. Necessary and sufficient condition in terms of maximum principle has been proven. In addition, transversation conditions have been found to establish the transition points that are important for the switching systems. It is well known that, the feedback form of optimal control important for the Linear Quadrature Regulator problems. The solution of feedback problem directly relate with Riccati equations. Mentioned problem is solved for the stochastic differential equation with variable delay.
\end{abstract}

Keywords: Optimal control problem, stochastic differential equation with delay, switching systems, Riccati equations.

\section{Giriş}

Gerçek problemlerde karşılaştığımız sistemlerin dinamiğinin belirlenmesinde geçmiş bilgilerin önemi bir çok çalışmalarda öne sürülmektedir [1]. Bu sistemlerin ifade olunması için kullanılan matematiksel modeller içerisinde gecikmeli diferansiyel denklemler öne çıkmaktadır. Aynı zamanda bir çok biyoloji, tıp, ekonomi, mekanik vb. alanlarda karşılaştı̆̆ımız bir sıra sistemlerin tanımlanmasında belirsizlikleri göz önünde bulundurulmak zarureti duyulmaktadır [2-6]. Son zamanlarda yukarıda belirtilen iki önemli

*Sorumlu yazar: c.agayeva@alparslan.edu.tr

Geliş Tarihi: 15.10.2019, Kabul Tarihi: 16.06.2020 
özelliği içeren sistemlerin incelenmesi için yapılan araştırmalara sık sık rastlanmaktadır [7-9]. Son birkaç yıl içinde, özellikle hayat ve hayat dışı sigortalardan, matematiksel finanstan, sağlı, ekonomi ve teknik sistemlerden kaynaklanan stokastik optimizasyon problemleri önemli ölçüde gelişmiştir.

Rastgele etkilere ve zaman gecikmesine maruz kalan dinamik sistemlerin kontrol teorisi üzerine yapılan araştırmalara örnek olarak, Agayeva ve Allahverdiyeva [10], Balakrishnan [11], Chernousko ve Ananievski [12], Federico ve ark. [13], Larssen [14] çalışmaları gösterilebilir.

Doğrusal kontrol problemi, doğrusal stokastik diferansiyel denklemlerle ifade olunan ve belirli amaç fonksiyonunun en iyi değerinin bulunmasına dayanan ilk optimal kontrol problemi olarak bilinmektedir [15-18].

Bu problemlerin özel hali olan doğrusal karesel regülatör problemi, literatürdeki en çok çalışılan kontrol problemlerinden biri olmasına rağmen, düzgün ve düzgün olmayan doğrusal sistemler için hala açık kalan çeşitli optimal kontrol problemleri mevcuttur [19-21].

Düzgen olmayan hibrit sistemlerinin özel sınıfı olarak tanımlanan geçiş sistemleri, sürekli değişime sahip dinamiklerin modellenmesinde geniş ş̧ekilde kullanılmaktadır. Boukas [22], Kharatatishvili ve Tadumadze [23], Tadumadze ve Arsenashvili [24], Aghayeva [25], Abushov ve Aghayeva [26], Agayeva [27] ve benzer çalışmalarda bu sistemlerle bağlı çeşitli problemler ele alınmıştır.

$\mathrm{Bu}$ makalede değişken gecikmeli doğrusal stokastik diferansiyel denklemlerle ifade olunan sistemler için optimal kontrol problem ele alınmıştır. Sistemin sağ uç noktalarındaki kısıtlar da dikkate alınarak, optimal çözüm için gerek ve yeter koşul bulunmuştur.

\section{Materyal ve Metot}

\subsection{Problemin tanımlanması ve ön bilgiler}

Bu bölümde, çalışmada kullanılan notasyon ve tanımları belirledik. N pozitif bir sabit olsun; $R^{n}$ ile $\boldsymbol{n}$ boyutlu gerçek vektör uzayını, |.| ile Öklid normunu ve $\langle\cdot, \cdot\rangle$ ile skaler çarpımı işaretlenmiştir. Beklenen değer E olarak, $\{1, \ldots, \mathrm{S}\}$ tam sayılarının kümesi ise $\overline{1, s}$ olarak gösterilmiştir; ' simgesi türevi , * matrisler için transpose işlemini ifade etmektedir.

Varsayalım ki, $F_{t}^{l}=\bar{\sigma}\left(w_{q}^{l}, t_{l-1} \leq t \leq t_{l}\right), \quad l=\overline{1, s}$, bağımsız $w^{1}(t), w^{2}(t), \ldots, w^{s}(t)$ Wiener süreçlerinin ürettiği akımlar; $\left(\Omega, F^{l}, P\right), l=\overline{1, \text { sise }}\left\{F_{t}^{l}, t \in\left[t_{l-1}, t_{l}\right]\right\}$ akımlarının oluşturduğu olasılık uzayı olsun. $E \int_{a}^{b}|x(t, \omega)|^{2} d t<+\infty$ koşulunu sağlayan tüm öngörülebilir stokastik süreçler uzayını $L_{F^{l}}^{2}\left(a, b ; R^{n}\right)$ olarak gösterilmiştir. $R^{m}$ 'den $R^{n}$,e yansıyan tüm lineer dönüşümler ise $R^{m \times n}$ gibi gösterilmiştir.

$$
U=U^{1} \times U^{2} \times \ldots \times U^{s} \text { ve } \pi^{i}=\left(t_{0}, \ldots, t_{i}, x_{t_{1}}^{1}, \ldots, x_{t_{i}}^{i}, u^{1}, \ldots, u^{i}\right) \text { elemanlarından oluşan }
$$

kümeler $A_{i}=T^{i+1} \times \prod_{j=1}^{i} O_{j} \times \prod_{j=1}^{i} U^{j}$ olarak gösterilmiştir.

$O_{l} \subset R^{n_{l}}, Q_{l} \subset R^{m_{l}}$ açık kümeler; $T=[0, T]$ sonlu aralık ve $0=t_{0}<t_{1}<\ldots<t_{s}=T$ olsun.

Varsayalım ki, $\quad \mathrm{A}^{l}, B^{l}, L^{l}, Q^{l} \in R^{n_{l} \times n_{l}}, C^{l}, D^{l} \in R^{n_{l} \times m_{l}}, \Phi^{l}, K^{l} \in R^{n_{l}}$ sonlu deterministik matrisler, $G^{l}, M^{l} \in R^{n_{l} \times n_{l}}$ yar1-kesin positif, $N^{l} \in R^{m_{l} \times m_{l}}$ kesin positif deterministik matrislerdir. Aksi belirtilmediği sürece makalede $t=\left(t_{0}, t_{1}, \ldots, t_{s}\right), u=\left(u^{1}, u^{2}, \ldots, u^{s}\right) \quad, x=\left(x^{1}, x^{2}, \ldots, x^{s}\right)$ gösterimleri kullanılacaktır.

Aşağıdaki şekilde tanımlanan doğrusal geçiş sistemini ele alalım:

$$
\begin{gathered}
d x^{l}(t)=\left[A^{l} x^{l}(t)+B^{l} x^{l}(t-h(t))+C^{l} u^{l}(t)+D^{l} u^{l}(t-h(t))\right] d t+ \\
{\left[L^{l} x^{l}(t)+Q^{l} x^{l}(t-h(t))\right] d w^{l}(t),}
\end{gathered}
$$




$$
\begin{gathered}
x^{l+1}(t)=\Phi^{l} x^{l}(t)+K^{l}, t \in\left[t_{l}-h\left(t_{l}\right), t_{l}\right], l=\overline{1, s-1,} \\
x^{1}(t)=K^{0}, t \in\left[t_{0}-h\left(t_{0}\right), t_{0}\right], \\
u^{l}(t) \equiv u^{l}(t, \omega) \in U_{\partial}^{l} \equiv\left\{u^{l}(\cdot, \cdot) \in L_{F}^{2}\left(t_{l-1}, t_{l} ; R^{m_{l}}\right) \mid u^{l}(t, \cdot) \in U^{l} \subset R^{m_{l}}\right\} .
\end{gathered}
$$

Burada $U^{l}, l=\overline{1, s}$ boş olmayan sonlu kümelerdir. $U_{\partial}^{l}$ kümelerinin elemanlarını mümkün kontroller olarak tanımlayalım. Problem, (1)-(3) sisteminin (4) mümkün kontroller kümesinde tanımlanan çözümleri için,

$$
J(u)=E \sum_{l=1}^{s}\left\{\left\langle G^{l} x^{l}\left(t_{l}\right), x^{l}\left(t_{l}\right)\right\rangle+\int_{t_{l-1}}^{t_{l}}\left(\left\langle M^{l} x^{l}(t), x^{l}(t)\right\rangle+\left\langle N^{l} u^{l}(t), u^{l}(t)\right\rangle\right) d t\right\}
$$

amaç fonksiyonunu

$$
E\left\langle q^{s}\left(t_{s}\right), x^{s}\left(t_{s}\right)\right\rangle \in G
$$

koşulu altında minimize eden, $\left(x^{1}, x^{2}, \ldots, x^{s}, u^{1}, u^{2}, \ldots, u^{s}\right)$ optimal çözümün ve $t_{1}, t_{2}, \ldots, t_{s}$ geçiş dizisinin bulunmasından oluşmaktadır.

Burada $h(t)>0, t \in\left[t_{0}, t_{s}\right]$ sürekli diferansiyellenen deterministtik fonksiyon olup, $\frac{d h(t)}{d t}<1$ koşulunu sağlamaktadır, $G$ ise boş olmayan kapalı konveks kümedir.

Tanım 1.Eğer $x^{l}(t) \in O_{l}$ fonksiyonu $t \in\left[t_{l}-h\left(t_{l}\right), t_{l}\right]$ aralığında (2), (3) koşullarını tatmin ediyorsa ve nerdeyse tüm $\left[t_{l-1}, t_{l}\right]$ aralığında 1 olasilığıla mutlak sürekliyse, o zaman $\left\{x^{l}(t)=x^{l}\left(t, \pi^{l}\right), t \in\right.$ $\left.\left[t_{l}-h\left(t_{l}\right), t_{l}\right], l=\overline{1, s}\right\}$ fonksiyoneller kümesi (1) doğrusal stokastik diferansiyel denkleminin $\pi^{s} \in \mathrm{A}_{s}$ elemanına uygun gelen çözümüdür denir.

Tanım 2. $\left(x^{l}(t), u^{l}(t)\right), t \in\left[t_{l-1}-h\left(t_{l-1}\right), t_{l}\right], l=\overline{1, s}$ çiftleri (1)-(4) geçiş sisteminin çözümüyse ve (6) koşulunu sağlıyorsa, $\pi^{s} \in A_{s}$ 'ye mümkün eleman denir.

Tüm mümkün elemanlar kümesi $A_{s}^{0}$ olarak tanımlayalım.

Tanım 3. Eğer $\tilde{u}_{t}^{l}, l=\overline{1, s}$ mümkün kontrolleri ve (1)-(4), (6) sisteminin ona uygun gelen $\tilde{x}_{t}^{l}, l=\overline{1, s}$ çözümleri varsa ki, $\left(\tilde{x}_{t}^{l}, \tilde{u}_{t}^{l}\right)$ çiftleri (5) fonksiyonunu minimize ediyor, $\tilde{\pi}^{s} \in A_{s}^{0}$ elemanına (1)-(6) probleminin optimal çözümü denir.

\subsection{Doğrusal geçiş sistemleri için optimallık koşulu}

Ele alınan (1)-(6) kısıtlı stokastik optimal kontrol probleminin çözümü için önce (1)-(5) kısıtsız optimizasyon probleminin incelenmesi gerekiyor. Aşağıdaki teorem stokastik geçiş sistemleri için ele alınan (1)-(5) doğrusal regülatör problemi için gerek ve yeter koşulu ifade ediyor. Teoremin ispatı, Agayeva [27] çalışmasındaki yaklaşımlara benzer yöntemler izlenmekle, Agayeva ve Takan [28] makalesinde verilmiştir.

Teorem 1 ([28]). Varsayalım ki, $\left(\psi^{l}(t), \beta^{l}(t)\right) \in L_{F}^{2}\left(t_{l-1}, t_{l} ; R^{n_{l}}\right) \times L_{F}^{2}\left(t_{l-1}, t_{l} ; R^{n_{l} x n_{l}}\right)$ rassal süreçleri 


$$
\left\{\begin{array}{c}
d \psi^{l}(t)=-\left[A^{l^{*}} \psi^{l}(t)+B^{l^{*}} \psi^{l}(r(t)) r^{\prime}(t)+L^{l^{*}} \beta^{l}(t)+{ }^{\prime} Q^{l^{*}} \beta^{l}(r(t)) r^{\prime}(t)-M^{l} x^{l}(t)\right] d t \\
+\beta^{l}(t) d w^{l}(t), \quad t \in\left[t_{l-1}, t_{l}-h\left(t_{l}\right)\right), \quad l=\overline{1, s} \\
d \psi^{l}(t)=-\left[A_{t}^{l^{*}} \psi^{l}(t)+B^{l+1^{*}} \psi^{l+1}(t) \Phi^{l}+L_{t}^{l^{*}} \beta^{l}(t)+Q^{l+1^{*}} \beta^{l+1}(t) \Phi^{l}-M^{l} x^{l}(t)\right] d t \\
+\beta^{l}(t) d w^{l}(t), \quad t \in\left[t_{l}-h\left(t_{l}\right), t_{l}\right), \quad l=\overline{1, s} \\
\psi^{l}\left(t_{l}\right)=-G^{l} x^{l}\left(t_{l}\right)+\Phi^{l *} \psi^{l+1}\left(t_{l}\right), l=\overline{1, s-1} \\
\psi^{s}\left(t_{s}\right)=-G^{s} x^{s}\left(t_{s}\right) ;
\end{array}\right.
$$

stokastik denklemler sisteminin çözümleridir ve burada $\tau=r(\tau)$ fonksiyonu $\tau=t-h(t)$ denkleminin çözümüdür. $\mathrm{Bu}$ zaman $\pi^{s}=\left(t_{0}, \ldots, t_{s}, x_{t}^{1}, \ldots, x_{t}^{S}, u^{1}, \ldots, u^{s}\right)$ elemanının (1)-(5) probleminin optimal çözümü olması için gerek ve yeter koşul aşağıdakilerdir:

a) $u^{l}(t), l=\overline{1, s}$ optimal kontrol adayları aşağıdaki gösterimle belirleniyor:

$$
\left\{\begin{array}{c}
N^{l *} u^{l}(t)=C^{l *} \psi^{l}(t)+D^{l *} \psi^{l}(r(t)) r^{\prime}(t), t \in\left[t_{l-1}, t_{l}-h\left(t_{l}\right)\right) \\
N^{l *} u^{l}(t)=C^{l *} \psi^{l}(t), t \in\left[t_{l}-h\left(t_{l}\right), t_{l}\right]
\end{array}\right.
$$

b) Tüm $t_{1}, t_{2}, \ldots, t_{s-1}$ noktaları için:

$$
\begin{gathered}
\psi^{l}\left(t_{l}\right)\left[A^{l} x^{l}\left(t_{l}\right)+B^{l} x^{l}\left(t_{l}-h\left(t_{l}\right)\right)+C^{l} u^{l}\left(t_{l}\right)+D^{l} u^{l}\left(t_{l}-h\left(t_{l}\right)\right)\right]+\beta^{l}\left(t_{l}\right)\left[L^{l} x^{l}\left(t_{l}\right)+\right. \\
\left.Q^{l} x^{l}\left(t_{l}-h\left(t_{l}\right)\right)\right]=\psi^{l+1}\left(t_{l}\right)\left[A^{l+1} x^{l+1}\left(t_{l}\right)+B^{l+1} x^{l+1}\left(r\left(t_{l}\right)\right) r^{\prime}\left(t_{l}\right)+\right. \\
\left.C^{l+1} u^{l+1}\left(t_{l}\right)+D^{l+1} u^{l+1}\left(r\left(t_{l}\right)\right) r^{\prime}\left(t_{l}\right)\right]+\beta^{l+1}\left(t_{l}\right)\left[L^{l+1} x^{l+1}\left(t_{l}\right)+Q^{l+1} x^{l+1}\left(r\left(t_{l}\right)\right) r^{\prime}\left(t_{l}\right)\right]
\end{gathered}
$$

geçiş kuralları sağlanmaktadır.

Şimdi Teorem 1'e dayanarak (1)-(6) eşitlikleriyle tanımlanan kısıtlı stokastik regülatör problemini inceleyelim. Kullanacağımız yaklaşımda ihtiyacımız olacak Ekeland Varyasyon Prensibini tanımlayalım.

Ekeland varyasyon prensibi (Ekeland, [29]). $\boldsymbol{K}$ tam metrik uzay, $f: \boldsymbol{K} \rightarrow R \cup\{+\infty\}$ ise alttan sinırlı ve yarıürekli fonksiyon olsun. $f(x)$ fonksiyonu $x_{0}$ noktasında positif $\varepsilon$ değeri için

$$
f\left(x_{0}\right) \leq \inf f(x)+\varepsilon
$$

koşulunu sağlasın. Bu zaman keyfi $\lambda>0$ için $\exists \bar{x} \in \boldsymbol{K}$ noktası var ki,

1) $f(\bar{x}) \leq f\left(x_{0}\right)$

2) $d\left(x_{0}, \bar{x}\right) \leq \lambda$,

3) $\forall x \neq \bar{x}$ için

doğru oluyor.

Teorem 2. Varsayalım ki, $\left(\psi^{l}(t), \beta^{l}(t)\right) \in L_{F^{l}}^{2}\left(t_{l-1}, t_{l} ; R^{n_{l}}\right) \times L_{F}^{2}\left(t_{l-1}, t_{l} ; R^{n_{l} x n_{l}}\right)$ rassal süreçleri ve $\lambda=\left(\lambda_{0}^{S}, \lambda_{1}^{1}, \ldots, \lambda_{1}^{s}\right), \lambda_{0}^{S} \leq 0$ vektörü:

$$
\left\{\begin{array}{c}
d \psi^{l}(t)=-\left[A^{l^{*}} \psi^{l}(t)+B^{l^{*}} \psi^{l}(r(t)) r^{\prime}(t)+L^{l^{*}} \beta^{l}(t)+{ }^{\prime} Q^{l^{*}} \beta^{l}(r(t)) r^{\prime}(t)-M^{l} x^{l}(t)\right] d t \\
+\beta^{l}(t) d w^{l}(t), \quad t \in\left[t_{l-1}, t_{l}-h\left(t_{l}\right)\right), \quad l=\overline{1, s} \\
d \psi^{l}(t)=-\left[A_{t}^{l^{*}} \psi^{l}(t)+B^{l+1^{*}} \psi^{l+1}(t) \Phi^{l}+L_{t}^{l^{*}} \beta^{l}(t)+Q^{l+1^{*}} \beta^{l+1}(t) \Phi^{l}-M^{l} x^{l}(t)\right] d t \\
+\beta^{l}(t) d w^{l}(t), \quad t \in\left[t_{l}-h\left(t_{l}\right), t_{l}\right), \quad l=\overline{1, s} \\
\psi^{l}\left(t_{l}\right)=-\lambda_{1}^{l} G^{l} x^{l}\left(t_{l}\right)+\Phi^{l *} \psi^{l+1}\left(t_{l}\right), l=\overline{1, s-1} \\
\psi^{s}\left(t_{s}\right)=-\lambda_{1}^{s} G^{s} x^{s}\left(t_{s}\right)-\lambda_{0}^{s} q^{s}\left(t_{s}\right)
\end{array}\right.
$$


eşlenik stokastik denklemler sisteminin çözümü olsun. Bu durumda;

$\pi^{s}=\left(t_{0}, \ldots, t_{s}, x_{t}^{1}, \ldots, x_{t}^{s}, u^{1}, \ldots, u^{s}\right)$ elemanının (1)-(6) probleminin optimal çözümü olması için gerek ve yeter koşul, (8), $t_{1}, \ldots, t_{s-1}$ noktalarında (9) ve $t_{s}$ noktasında ise:

$$
\begin{aligned}
& \psi^{s}\left(t_{s}\right)\left[A^{s} x^{s}\left(t_{s}\right)+B^{s} x^{s}\left(t_{s}-h\left(t_{s}\right)\right)+C^{s} u^{s}\left(t_{s}\right)+D^{s} u^{s}\left(t_{s}-h\left(t_{s}\right)\right)\right] \\
& \quad+\beta^{s}\left(t_{s}\right)\left[L^{s} x^{s}\left(t_{s}\right)+Q^{s} x^{s}\left(t_{s}-h\left(t_{s}\right)\right)\right]+q_{t}^{s}\left(t_{s}\right)=0
\end{aligned}
$$

eşitliklerinin doğru olmasıdır. Burada $q_{t}^{S}\left(t_{s}\right)$ ile $q^{s}(t)$ fonksiyonunun $t_{s}$ noktasındaki türevi gösterilmektedir.

İspat. Her bir pozitif $\boldsymbol{j}$ tam sayısı için,

$$
I_{j}(u)=\min _{(c, y) \in \varepsilon} \sqrt{\sum_{l=1}^{s}\left|c^{l}-1 / j-E S^{l}(x, u, t)\right|^{2}+\left|y-E\left\langle q^{s}\left(t_{s}\right), x^{s}\left(t_{s}\right)\right\rangle\right|^{2}}
$$

şeklinde yaklaşık fonksiyonu tanımlayalım. Burada $\varepsilon=\left\{c: c \leq J^{0}, y \in G\right\}, \quad c=c^{1}+\ldots+c^{s}$, $S^{l}(x, u, t)=\left\langle G^{l} x^{l}\left(t_{l}\right), x^{l}\left(t_{l}\right)\right\rangle+\int_{t_{l-1}}^{t_{l}}\left(\left\langle M^{l} x^{l}(t), x^{l}(t)\right\rangle+\left\langle N^{l} u^{l}(t), u^{l}(t)\right\rangle\right) d t \quad$ ve $\quad J^{0} \quad$ ise (1)-(5) probleminde fonksiyonelin minimal değerini ifade ediyor. Varsayalım ki, $V \equiv\left(V^{1}, \ldots, V^{r}\right)$, nerede ki, $V^{k} \equiv\left(U^{k}, d\right), \quad d\left(u^{k}, v^{k}\right)=(l \otimes P)\left\{(t, \omega) \in\left[t_{k-1}, t_{k}\right] \times \Omega: v_{t}^{k} \neq u_{t}^{k}\right\}$ metriğine göre tanımlanan kontroller uzayıdır. Ekeland Varyasyon Prensibine göre, $\exists u_{t}^{l, j}: d\left(u_{t}^{l, j}, u_{t}^{l}\right) \leq \sqrt{\varepsilon_{j}^{l}}$ kontrolü var ki, $\forall u_{t}^{l} \in V^{l}$ için aşağıdaki eşitsizlik sağlanmaktadır:

$I_{j}\left(u^{j}\right) \leq I_{j}(u)+\frac{1}{j} d\left(u^{j}, u\right), d\left(u^{j}, u\right)=\sum_{l=1}^{s} d\left(u^{l, j}, u^{l}\right)$.

Böylece, kısitsız

$$
\left\{\begin{array}{c}
J_{j}(u)=I_{j}\left(u^{j}\right)+E \sum_{l=1}^{s} \sqrt{\varepsilon_{j}^{l}} \int_{t_{l-1}}^{t_{l}} \delta^{l}\left(u_{t}^{l}, u_{t}^{l, j}\right) d t \rightarrow \min \\
d x_{t}^{l, j}=\left[A^{l} x^{l, j}(t)+B^{l} x^{l, j}(t-h(t))+C^{l} u^{l, j}(t)+D^{l} u^{l, j}(t-h(t))\right] d t+ \\
{\left[L^{l} x^{l, j}(t)+Q^{l} x^{l, j}(t-h(t))\right] d w^{l}(t), t \in\left(t_{l-1}, t_{l}\right]} \\
x_{t}^{1+1, j}=\Phi^{l} x^{l, j}(t)+K^{l}, t \in\left[t_{l}-h\left(t_{l}\right), t_{l}\right], \quad l=1, \ldots, s-1, \\
x_{t}^{1, j}=K^{0}, t \in\left[t_{0}-h\left(t_{0}\right), t_{0}\right], \quad u_{t}^{l} \in U_{\partial}^{l}
\end{array}\right.
$$

doğrusal stokastik kontrol probleminin çözümünü $\left(t_{0}, t_{1}, \ldots, t_{r}, x_{t}^{1, j}, \ldots, x_{t}^{r, j}, u_{t}^{1, j}, \ldots, u_{t}^{r, j}\right)$ olarak gösterelim.

Varsayalım ki, $\psi_{t}^{l, j} \in L_{F}^{2}\left(t_{l-1}, t_{l} ; R^{n_{l}}\right), \beta_{t}^{l, j} \in L_{F}^{2}\left(t_{l-1}, t_{l} ; R^{n_{l} \times n_{l}}\right)$ rassal süreçleri ve sıfır olmayan $\left(\lambda_{0}^{s, j}, \lambda_{1}^{1, j}, \ldots, \lambda_{1}^{s, j}\right)$ vektörleri var ki, 


$$
\left\{\begin{array}{c}
d \psi^{l, j}(t)=-\left[A^{l^{*}} \psi^{l, j}(t)+B^{l^{*}} \psi^{l, j}(r(t)) r^{\prime}(t)+L^{l^{*}} \beta^{l, j}(t)+{ }^{\prime} Q^{l^{*}} \beta^{l, j}(r(t)) r^{\prime}(t)-\right. \\
\left.-M^{l} x^{l, j}(t)\right] d t d x_{t}^{l, j}=+\beta^{l, j}(t) d w^{l}(t), \quad t \in\left[t_{l-1}, t_{l}-h\left(t_{l}\right)\right), \quad l=\overline{1, s} \\
d \psi^{l, j}(t)=-\left[A^{l^{*}} \psi^{l, j}(t)+B^{l+1^{*}} \psi^{l+1, j}(t) \Phi^{l}+L_{t}^{l^{*}} \beta^{l, j}(t)+Q^{l+1^{*}} \beta^{l+1, j}(t) \Phi^{l}\right. \\
\left.-M^{l} x^{l, j}(t)\right] d t+\beta^{l, j}(t) d w^{l}(t), \quad t \in\left[t_{l}-h\left(t_{l}\right), t_{l}\right), \quad l=\overline{1, s} \\
\psi^{l, j}\left(t_{l}\right)=-\lambda_{1}^{l, j} G^{l} x^{l, j}\left(t_{l}\right)+\Phi^{l *} \psi^{l+1, j}\left(t_{l}\right), l=\overline{1, s-1} \\
\psi^{s, j}\left(t_{s}\right)=-\lambda_{1}^{s, j} G^{s} x^{s, j}\left(t_{s}\right)-\lambda_{0}^{s, j} q^{s}\left(t_{s}\right) ;
\end{array}\right.
$$

sisteminin çözümüdürler. $\left(\lambda_{0}^{s, j}, \lambda_{1}^{1, j}, \ldots, \lambda_{1}^{s, j}\right)$ vektörü

$$
\left(-y+E q^{s} x^{s, j}\left(t_{s}\right),-c^{1}+\varepsilon_{j}^{1}+E S^{1}\left(x^{1}, u^{1}, t\right), \ldots,-c^{s}+\varepsilon_{j}^{s}+E S^{s}\left(x^{s}, u^{s}, t\right)\right) / J_{j}^{0}
$$

ifadesiyle ve

$$
J_{j}^{0}=\left(\sum_{l=1}^{s}\left|c^{l}-1 / j-E S^{l}\left(x^{j}, u^{j}, t\right)\right|^{2}+\left|y-E\left\langle q^{s}\left(t_{s}\right), x^{s, j}\left(t_{s}\right)\right\rangle\right|^{2}\right)^{\frac{1}{2}} .
$$

olarak tanımlanmışlar.

$\mathrm{Bu}$ durumda Teorem 1'e dayanarak, aşağıdaki sonuca varmış oluyoruz:

$u^{l, j}(t), l=\overline{1, s}$ elemanları (12) kontrol probleminin optimal çözümü olması için gerek ve yeter koşul,

$$
\left\{\begin{array}{c}
N^{l *} u^{l, j}(t)=C^{l *} \psi^{l, j}(t)+D^{l *} \psi^{l, j}(r(t)) r^{\prime}(t), t \in\left[t_{l-1}, t_{l}-h\left(t_{l}\right)\right) \\
N^{l *} u^{l, j}(t)=C^{l *} \psi^{l, j}(t), \quad t \in\left[t_{l}-h\left(t_{l}\right), t_{l}\right]
\end{array}\right.
$$

sağlanmasıdır. Bunun yanı sıra , tüm $t_{1}, t_{2}, \ldots, t_{s-1}$ noktaları için:

$$
\begin{gathered}
\psi^{l, j}\left(t_{l}\right)\left[A^{l} x^{l, j}\left(t_{l}\right)+B^{l} x^{l, j}\left(t_{l}-h\left(t_{l}\right)\right)+C^{l} u^{l, j}\left(t_{l}\right)+D^{l} u^{l, j}\left(t_{l}-h\left(t_{l}\right)\right)\right] \\
+\beta^{l, j}\left(t_{l}\right)\left[L^{l} x^{l, j}\left(t_{l}\right)+Q^{l} x^{l, j}\left(t_{l}-h\left(t_{l}\right)\right)\right]= \\
\psi^{l+1, j}\left(t_{l}\right)\left[A^{l+1} x^{l+1, j}\left(t_{l}\right)+B^{l+1} x^{l+1, j}\left(r\left(t_{l}\right)\right) r^{\prime}\left(t_{l}\right)+C^{l+1} u^{l+1, j}\left(t_{l}\right) u^{l+1, j}\left(r\left(t_{l}\right)\right) r^{\prime}\left(t_{l}\right)\right] \\
+\beta^{l+1, j}\left(t_{l}\right)\left[L^{l+1} x^{l+1, j}\left(t_{l}\right)+Q^{l+1} x^{l+1, j}\left(r\left(t_{l}\right)\right) r^{\prime}\left(t_{l}\right)\right] ;
\end{gathered}
$$

ve $t_{s}$ noktasinda ise:

$$
\begin{gathered}
\psi^{s, j}\left(t_{s}\right)\left[A^{s} x^{s, j}\left(t_{s}\right)+B^{s} x^{s, j}\left(t_{s}-h\left(t_{s}\right)\right)+C^{s} u^{s, j}\left(t_{s}\right)+D^{s} u^{s, j}\left(t_{s}-h\left(t_{s}\right)\right.\right. \\
+\beta^{s, j}\left(t_{s}\right)\left[L^{s} x^{s, j}\left(t_{s}\right)+Q^{s} x^{s, j}\left(t_{s}-h\left(t_{s}\right)\right)\right]+q_{t}^{s}\left(t_{s}\right)=0
\end{gathered}
$$

geçiş koşullarını sağlanmış oluyor.

Eşitlik (14)'den $\left|\lambda_{0}^{s, j}\right|^{2}+\sum_{l=1}^{s}\left\langle\lambda_{1}^{l, j}, \lambda_{1}^{l, j}\right\rangle=1$ olduğunu ve $\left(\lambda_{0}^{s, j}, \lambda_{1}^{1, j}, \ldots, \lambda_{1}^{s, j}\right) \rightarrow\left(\lambda_{0}^{s}, \lambda_{1}^{1}, \ldots, \lambda_{1}^{s}\right)$ eğer $j \rightarrow \infty$ yakınsaklığını elde etmiş oluruz.

Aynı zamanda $I_{j}(u)$ konveks fonksiyonunun $\left(E S^{1, j}(x, u, t), \ldots, E S^{s, j}(x, u, t), E\left\langle q^{s}\left(t_{s}\right), x^{s, j}\left(t_{s}\right)\right\rangle\right)$ noktasında sonlu diferansiyelinin varlığından $\lambda_{1}^{l} \leq 0, l=1, \ldots, s$ olduğunu ve $E\left\langle q^{s}\left(t_{s}\right), x^{s}\left(t_{s}\right)\right\rangle$ noktasında $\lambda_{0}^{s}$ 'ın $\mathbf{G}$ kümesi için normal oluşturduğunu kanıtlamış oluyoruz. Böylece,

$\psi^{l, j}\left(t_{l}\right)=-\lambda_{1}^{l, j} G^{l} x^{l, j}\left(t_{l}\right)+\Phi^{l *} \psi^{l+1, j}\left(t_{l}\right) \quad$ ve $\quad \psi^{s, j}\left(t_{s}\right)=-\lambda_{0}^{s, j} q^{s}\left(t_{s}\right)-\lambda_{1}^{s, j} G^{s} x^{s, j}\left(t_{s}\right)$ ifadelerinden $\psi^{l}\left(t_{l}\right)=-\lambda_{1}^{l} G^{l} x^{l}\left(t_{l}\right)+\Phi^{l *} \psi^{l+1}\left(t_{l}\right)$ ve $\psi^{s}\left(t_{s}\right)=-\lambda_{0}^{s} q^{s}\left(t_{s}\right)-\lambda_{1}^{s} G^{s} x^{s}\left(t_{s}\right)$ eşitlikleri elde ediliyor. Bununla da, $\psi_{t}^{l, j}, \beta_{t}^{l, j}$ dizisinin zayıf limitinin $\psi_{t}^{l}, \beta_{t}^{l}$ olduğu sağlanmış oluyor. Nihayet, (15)-(17) ifadelerinde limit alınarak, (8),(9),(11) eşitliklerini doğrulamış oluyoruz. 


\subsection{Gecikmeli stokastik sistemler için Riccati denklemi}

Doğrusal Karesel Regülatör problemlerinin önemli yönü optimal kontrolün geri dönüşüm şeklinde ifadesinin oluna bilinmesidir. Bir başa Riccati denklemleriyle ilişkili olan problem, bu çalışmada değişken gecikmeli stokastik sistemler için Teorem 2'de eşitlik (8) ile ifade olunan optimal kontrolü ileri diferansiyel denklemlerle formüle etmekten ibarettir. Ele alınan regülatör probleminde geri dönüşüm förmülü için, (10) stokastik eklenik denkleminin çözümü faz degişkenlerinin doğrusal fonksiyonu olarak 1 olasılıkla aşağıdaki gibi aranacaktır:

$$
\psi^{l}(t)=-p^{l}(t) x^{l}(t), l=\overline{1, . S}
$$

Amacımız, bilinmeyen $p^{l}(t), l=1, \ldots, s$ rassal süreçlerinin şeklini $d p^{l}(t)=\alpha^{l}(t) d t+\gamma^{l}(t) d w^{l}(t), l=1, \ldots, s$

şeklinde tanımlayıp, uygun Ricati denklemlerini bulmaktadır. tokastik süreçlerin zamana göre diferansiyelinin bulunması için bilinen İto formülüne [3], dayanarak (18) eşitliğinden ve (1) denkleminden:

$$
\begin{gathered}
-d \psi^{l}(t)=d p^{l}(t) x^{l}(t)+p^{l}(t) d x^{l}(t)+\gamma^{l}(t) L^{l} x^{l}(t)+\gamma^{l}(t) Q^{l} x^{l}(t-h(t)) d t= \\
d p^{l}(t) x^{l}(t)+p_{t}^{l}\left[A^{l} x^{l}(t)+B^{l} x^{l}(t-h(t))+C^{l}(t) u^{l}(t)+D^{l}(t) u^{l}(t-h(t))\right] d t+ \\
\gamma^{l}(t)\left[L^{l} x^{l}(t)+Q^{l} x^{l}(t-h(t))\right] d t+p^{l}(t)\left[L^{l} x^{l}(t)+Q^{l} x^{l}(t-h(t))\right] d w^{l}(t) .
\end{gathered}
$$

Her bir $l=\overline{1, s}$ için (10) denklemine göre:

$$
\begin{aligned}
& \int_{t_{l-1}}^{t_{l}-h}\left[A^{l^{*}} \psi^{l}(t)+D^{l^{*}} \beta^{l}(t)+B^{l^{*}} \psi^{l}(r(t)) r^{\prime}(t)-M^{l} x^{l}(t)\right] \chi_{l} d t+\int_{t_{t_{l}}}^{t_{l}}\left[A^{l^{*}} \psi^{l}(t)+D^{l^{*}} \beta^{l}(t)\right. \\
& \left.+B^{l+1^{*}} \psi^{l+1}(r(t)) r^{\prime}(t) \Phi^{l}-M^{l} x^{l}(t)\right]\left(1-\chi_{l}\right) d t-\int_{t_{l-1}}^{t_{l}} \beta^{l}(t) d w^{l}(t)= \\
& \int_{t_{l-1}}^{t_{l}}\left[\alpha^{l}(t) x^{l}(t)+p^{l}(t) A^{l} x^{l}(t)+p^{l}(t) B^{l} x^{l}(t-h(t))+p^{l}(t) C^{l} u^{l}(t)+p^{l}(t) D^{l} u^{l}(t-h(t))\right. \\
& \begin{array}{c}
\left.\left.+\gamma^{l}(t) L^{l} x^{l}(t)+\gamma^{l}(t) Q^{l} x^{l}(t) h(t)\right)\right] d t \\
+\int_{t_{l-1}}^{t_{l}}\left[\gamma^{l}(t) x^{l}(t)+p^{l}(t) L^{l} x^{l}(t)+p^{l}(t) Q^{l} x^{l}(t-h(t))\right] d w^{l}(t)
\end{array}
\end{aligned}
$$

elde etmiş oluruz. Burada $\chi_{l}$ ise $\left[t_{l-1}, t_{l}-h\left(t_{l}\right)\right]$ aralığının gösterge fonksiyonudur.

Son işlemleri dikkate alarak, optimal kontrol için

$$
\left\{\begin{array}{c}
N^{l *} u^{l, j}(t)=C^{l *} p^{l}(t)+D^{l *} p^{l}(t-h(t)), x^{l}(t-h(t)), t \in\left[t_{l-1}, t_{l}-h\left(t_{l}\right)\right) \\
N^{l *} u^{l}(t)=C^{l *} p^{l}(t), x^{l}(t) \quad t \in\left[t_{l}-h\left(t_{l}\right), t_{l}\right]
\end{array}\right.
$$

şeklinde geri dönüşüm ifadesini elde etmiş oluruz ki, buradaki $\left(p_{t}^{l}, \gamma_{t}^{l}\right)$ rassal süreçleri aşağıdaki Riccati denkleminin çözümüdür:

$$
\begin{gathered}
d p^{l}(t)=-\left[p^{l}(t) A^{l}+A^{l^{*}} p^{l}(t)+\gamma^{l}(t) L^{l}+L^{l^{*}} \gamma^{l}(t)+L_{t}^{l^{*}} p^{l}(t) L^{l}+p^{l}(r(t)) r^{\prime}(t) B^{l}+\right. \\
\left.B^{l^{*}} p^{l}(r(t)) r^{\prime}(t)+\gamma^{l}(r(t)) r^{\prime}(t) Q^{l}+Q^{l^{*}} \gamma^{l}(r(t)) r^{\prime}(t)+M^{l}-p^{l}(t) C^{l}\left(N^{l^{*}}\right)^{-1} C^{l^{*}} p^{l}(t)\right] d t \\
+\gamma^{l}(t) d w^{l}(t),\left[t_{l-1}, t_{l}-h\right] .
\end{gathered}
$$




$$
\begin{gathered}
d p^{l}(t)=-\left[p^{l}(t) A^{l}+A^{l^{*}} p^{l}(t)+\gamma^{l}(t) L^{l}+L^{l^{*}} \gamma^{l}(t)+L_{t}^{l^{*}} p^{l}(t) L^{l}+M^{l}-\right. \\
\left.p^{l}(t) C^{l}\left(N^{l^{*}}\right)^{-1} C^{l^{*}} p^{l}(t)\right] d t+\gamma^{l}(t) d w^{l}(t),\left(t_{l}-h\left(t_{l}\right), t_{l}\right] .
\end{gathered}
$$

\section{Sonuç ve Öneriler}

Bu çalışmada, düzgen olmayan rassal sistemler için değişken gecikmeli optimal kontrol problemleri ele alınmıştır. Optimal çözüm için gerek ve yeter koşulu ifade eden Teorem 2, incelenen regülatör probleminde durum ve kontrol değişkenleri arasında olan geri dönüşümü göstermektedir. Bu bilgiye dayanarak çalı̧̧mada optimal kontrol için açık analitik ifade alınmıştır. Makalede ele alınan problem Agayeva ve Abushov [30] çalışmasının düzgen olmayan sistemler, Ağayeva ve Takan [28] makalesindeki modelin gecikmeli kontrol içeren durum için geliştirilmiş hali olarak kabul oluna bilir. Belirtilen çalışmalardaki sonuçlar ise, bu makaledeki (6), (9), (10) ve (11) eşitlikleriyle tanımlanan optimallık koşullarının özel durumu olarak yorumlana bilir.

\section{Yazarların Katkısı}

Çalışmada tüm katkı yazara aittir.

\section{Çıkar Çatışması Beyanı}

Yazarlar arasında herhangi bir çıkar çatışması bulunmamaktadır.

\section{Araştırma ve Yayın Etiği Beyanı}

Yapılan çalışmada araştırma ve yayın etiğine uyulmuştur.

\section{Kaynaklar}

[1] Kolmanovsky V., Myshkis A. 1992. Applied Theory of Functional Differential Equations. Kluwer Academic Publishers, Dordrecht, 1-234.

[2] Anderson B., Ilchmann A., Wirth F. 2013. Stabilizability of time-varying linear systems. Systems and Control Letters, 62: 747-755.

[3] Gikhman I., Skorokhod A. 1972. Stochastic Differential Equations. Springer, Berlin, 1-356.

[4] Hoek J., Elliott R. 2012. American option prices in a Markov chain model. Applied Stochastic Models in Business and Industry, 28: 35-39.

[5] Mao X. 2007. Stochastic Differential Equations and Their Applications. Horwood Publication House, Chichester, 440.

[6] Shen H., Xu Sh., Song X., Luo J. 2009. Delay-dependent robust stabilization for uncertain stochastic switching system with distributed delays. Asian Journal of Control, 5: 527-535.

[7] Chojnowska-Michalik A. 1978. Representation theorem for general stochastic delay equations. Bull. Acad. Polon. Sci. Ser. Sci. Math. Astronom. Phys., 7: 635-642 .

[8] Elsanosi I., Øksendal B., Sulem A. 2000. Some solvable stochastic control problems with delay. Stochastics and Stochastics Reports, 1-2: 69-89.

[9] Kohlmann M., Zhou X. 2000. Relationship between backward stochastic differential equations and stochastic controls: A linear-quadratic approach. SIAM, Journal on Control and Optimization, 38: 1392-1407.

[10] Agayeva C., Allahverdiyeva J. 2007. On one stochastic optimal control problem with variable delays. Theory of stochastic processes, 13: 3-11.

[11] Balakrishnan A. 1975. A note on the structure of optimal stochastic control. Applied Mathematics and Optimization, 1: 87-94.

[12] Chernousko F., Ananievski I., Reshmin S. 2008. Control of Nonlinear Dynamical Systems: Methods and Applications (Communication and Control Engineering). Springer, Berlin, 1-396. 
[13] Federico S., Golds B., Gozzi F. 2011. HJB equations for the optimal control of differential with delays and state constraints, II: optimal feedbacks and approximations. SIAM, Journal on Control and Optimization, 49: 2378-2414.

[14] Larssen B. 2002. Dynamic programming in stochastic control of systems with delay. Stochastics and Stochastics Reports, 3-4: 651-673 .

[15] Kalman R. 1960. Contributions to the theory of optimal control. Boletin De La Sociedad Matematica Mexicana, 5: 102-119.

[16] Bellman R. 1955. Functional equations in the theory of dynamic programming, positivity and quasilinearity. Proceeding of National Academy of Science, USA, 41: 743-746.

[17] Bensoussan A., Delfour M., Mitter S. 1976. The linear quadratic optimal control problem for infinite dimensional systems over an infinite horizon; survey and examples. In: IEEE Conference on Decision and Control, December 1-3, Clearwater, USA, 746-751.

[18] Delfour M.C. 1986. The linear quadratic optimal control problem with delays in state and control variables: a state space approach. SIAM Journal on Control and Optimization, 24: 835-883.

[19] Ichikawa A. 1982. Quadratic control of evolution equations with delays in control. SIAM, Journal on Control and Optimization, 20: 645-668.

[20] Bismut J.M. 1976. Linear quadratic optimal stochastic control with random coefficients. SIAM, Journal on Control and Optimization, 14: 419-444.

[21] Wonham W. 1968. On a matrix Riccati equation of stochastic control. SIAM, Journal on Control and Optimization; 6: 312-326.

[22] Boukas E.-K. 2006. Stochastic Switching Systems: Analysis and Design. Birkhauer, Boston, 1376.

[23] Kharatatishvili G., Tadumadze T. 1997. The problem of optimal control for nonlinear systems with variable structure, delays and piecewise continuous prehistory. Memoirs on Differential Equations and Mathematical Physics, 11: 67-88.

[24] Tadumadze T., Arsenashvili A. 2008. Optimization of a delay variable structure system with mixed intermediate condition. Bulletin of the Georgian National Academy of Sciences, 2 (3): 2226.

[25] Aghayeva Ch. 2014. Necessary condition of optimality for stochastic switching systems with delay. In: International Conference on Mathematical Models and Methods in Applied Sciences, 23-25 September, Saint Petersburg, Russia, 54-58.

[26] Abushov Q., Aghayeva Ch. 2014. Stochastic maximum principle for the nonlinear optimal control problem of switching systems. Journal of Computational and Applied Mathematics, 259: 371-376.

[27] Agayeva Ch. 2016. Linear Quadratic Control Problem of Stochastic Switching Systems with Delay. Anadolu University Journal of Science and Technology-B, Theoretical Sciences, 4 (2): 52-58.

[28] Ağayeva Ç., Takan A.M. 2018. Restricted Optimal Control Problem for Stochastic Switching Systems with Variable Delay. Muş Alparslan Üniversitesi Fen Bilimleri Dergisi, 6 (2): 565-569.

[29] Ekeland I. 1974. On the variational principle. Journal Mathematical Analysis and Applications, 47: 324-353.

[30] Agayeva Ch., Abushov Q. 2005. Linear-square stochastic optimal control problem with variable delay on control and state,.Transactions ANAS, math.- ph. series, informatics and control problems, 3: 204-208. 